\title{
Sustentabilidade Ecológica pelo cálculo da Pegada Ecológica
}

\author{
Environmental Sustainability for the calculation of the Ecological Footprint
}

\author{
Eduardo Borges Lied'(*) \\ Adriele Prisca de Magalhães ${ }^{2}$ \\ Danilo Fernandes Soares ${ }^{3}$ \\ Ana Paula Trevisan ${ }^{4}$ \\ Camilo Freddy Mendoza Morejon ${ }^{5}$
}

\section{Resumo}

A metodologia da Pegada Ecológica (PE) consiste em calcular o tamanho da área bioprodutiva necessária para produzir recursos e assimilar as emissões de Dióxido de Carbono $\left(\mathrm{CO}_{2}\right)$ produzidas pela população em estudo. Nesse contexto, buscou-se quantificar o consumo médio dos principais recursos empregados no cotidiano da Universidade Tecnológica Federal do Paraná, campus Medianeira (UTFPR-MD) durante o ano de 2015. Os resultados indicam uma Pegada Ecológica total de 177,41 hectares, equivalente a uma área quase 15 vezes maior que o próprio campus. Constatou-se que o consumo de Alimentos e Infraestrutura e Edifícios, juntos correspondem a 84\% das emissões de $\mathrm{CO}_{2}$ e da composição da $\mathrm{PE}$. O consumo de energia elétrica resultou em uma área quase duas vezes maior que a área do campus, enquanto que o consumo de papel e de Mobilidade e Transporte, por sua vez, são os que apresentam menores impactos, com menos de $4 \%$ na composição total. A contribuição per capita da $\mathrm{PE}$ do campus corresponde a 0,0753 ha.hab ${ }^{-1} \cdot$ ano $^{-1}$. Esse valor está próximo à $\mathrm{PE}$ nacional que é 0,0725 gha.hab ${ }^{-1}$.ano ${ }^{-1}$. Comparado a outros câmpus universitários, a UTFPR-MD apresentou maior sustentabilidade, pois sua PE per capita é 63\% menor que a daUSP São Carlos e $56 \%$ menor que da USC. Embora seja um aspecto positivo, é fundamental diminuir ainda mais esse índice através da adoção de medidas mais sustentáveis junto

1 Dr.; Engenharia Química; Universidade Estadual do Oeste do Paraná, UNIOESTE, Brasil; Professor Adjunto da Universidade Tecnológica Federal do Paraná, Câmpus Medianeira; Endereço: Universidade Tecnológica Federal do Paraná, Campus Medianeira. Avenida Brasil, 4232, Parque Independência. CEP: 85884-000 - Medianeira, PR - Brasil; E-mail: dudulied@hotmail.com

2 Mes.; Ecologia de Biomas Tropicais; Universidade Federal de Ouro Preto, UFOP, Brasil; Professora e Coordenadora do Laboratório de Biologia na Universidade do Estado de Minas Gerais, UEMG; Endereço: Universidade do Estado de inas Gerais, Faculdade de Engenharia - FaEnge. Avenida Brasília, Baú. CEP: 35930314 - João Monlevade, MG - Brasil; E-mail: adrielemagalhaes@gmail.com

3 Engenheiro Ambiental; Universidade Tecnológica Federal do Paraná, Campus Medianeira, UTFPR, Brasil; Endereço: Universidade Tecnológica Federal do Paraná, UTFPR, Brasil; E-mail: danilofsoares@hotmail.com

4 Dra.; Engenharia Agrícola; Universidade Estadual do Oeste do Paraná, UNIOESTE, Brasil; Professor titular na União Educacional de Cascavel, UNIVEL, Brasil; Endereço: Av. Tito Muffato, 2317 - Santa Cruz, Cascavel - PR, 85806-080; E-mail: anapaullatrevisan@gmail.com

5 Dr.; Engenharia Mecânica; Universidade Federal do Rio de Janeiro, COPPE/UFRJ, Brasil; professor associado da UNIOESTE e atua nos cursos de Graduação, Mestrado e Doutorado em Engenharia Química, Mestrado em Ciências Ambientais e Mestrado em Rede Nacional em Propriedade Industrial e Transferência de Tecnologia para a Inovação (PROFNIT); Endereço: Universidade Estadual do Oeste do Paraná, Centro de Engenharias e Ciências Exatas, Engenharia Química. Rua da Faculdade, 645, Jardim La Salle; CEP: 85903-000 - Toledo, PR - Brasil; E-mail:

\begin{tabular}{llllll}
\hline Ambiência & Guarapuava (PR) v.I5 n.I & p. $240-256$ & Jan/Abr 20I9 & ISSN I808 - 025I
\end{tabular}


à população do campus. Ressalta-se, ainda, a importância da Pegada Ecológica como uma ferramenta de fácil aplicação e compreensão dos resultados, tornando-se peça fundamental na gestão ambiental do câmpus.

Palavras-chave: Indicadores de sustentabilidade; universidades; impacto ambiental.

\section{Abstract}

The methodology of Ecological Footprint (EF) is to calculate the size of bioproductive area needed to produce resources and assimilate the emissions of carbon dioxide $\left(\mathrm{CO}_{2}\right)$ produced by population. In this context, it sought to quantify the average consumption of the main resources used every day in Federal Technological University of Paraná, Campus Medianeira (UTFPR-MD) during the year 2015. The results indicate a total Ecological Footprint of 177.41 hectares, equivalent to an area about 15 times larger than the campus. It was found that the consumption of food and infrastructure and buildings, together account for $84 \%$ of $\mathrm{CO}_{2}$ emissions and $\mathrm{EF}$ composition. The electricity consumption resulted in an area twice the area of the campus. While the consumption of paper, mobility and transportation, those are with less impact, less than $4 \%$ of the total. The per capita contribution of EF at campus corresponds to 0.0753 $\mathrm{ha} /$ person/year, this score is near to EF national score which is $0.0725 \mathrm{ha} /$ person/year. Compared to other college campuses, UTFPR-MD showed greater sustainability because its EF per capita is 63\% lower than USP - São Carlos and 56\% lower than the USC. While it is a good point, it is crucial to reduce this rate through adopting more sustainable measures by the campus population. The Ecological Footprint is an easy application tool and to understand the results, becoming key player in environmental management of the campus.

Keywords: Sustainability indicators; universities; envinronmental impacts.

\section{Introdução}

A capacidade de suporte ou capacidade de carga do ambiente representa a capacidade máxima de impactos que um determinado ambiente suporta sem que seja afetada a sua resiliência. Ecologistas definem capacidade de suporte como o tamanho máximo da população de uma espécie que uma determinada área pode suportar sem reduzir sua habilidade de manter essa mesma espécie por um período indefinido de tempo (DAILY; EHRLICH, 1992).

A relação entre população, demanda e disponibilidade de recursos naturais determina a capacidade de suporte. Como definido por Maduro-Abreu et al. (2009, p. 75), "a capacidade de carga refere-se ao tamanho máximo estável de uma população, determinado pela quantidade de recursos disponíveis e pela demanda mínima individual". "Ela é definida pelo seu fator mais limitante e pode ser melhorada ou degradada pelas atividades humanas” (MONTEIRO, 2010, p. 26). 
Nesse contexto, ao se consubstanciarem essas definições, é possível concluir que a capacidade de suporte, depende principalmente de três critérios:

i. quantidade de indivíduos;

ii. demanda e disponibilidade de recursos naturais;

iii. espaço físico.

Segundo Pereira (2008, p. 23), "indicadores são parâmetros selecionados e considerados isoladamente ou combinados entre si, sendo especialmente úteis para refletir sobre determinadas condições dos sistemas em análise”. Todos os indicadores, independente do segmento de aplicação, possuem, em comum, a função básica de simplificar ou resumir informações relevantes sobre fenômenos complexos, facilitando a compreensão destes (ANDRADE; VAN BELLEN, 2006). "Indicadores têm como principal objetivo tornar compreensível um conjunto de fenômenos que não são facilmente percebidos” (LEITE; VIANA, 2001 p. 3).

Atualmente, existem várias metodologias que fazem uso de indicadores de sustentabilidade, como: Pressão - Estado - Resposta; Índice de Desenvolvimento Humano (IDH); Barômetro da Sustentabilidade; Análise Emergética; Pegada Ecológica; entre outros. Entretanto, até o momento não existe um indicador ambiental que seja universalmente aceito. Atualmente sobressaem-se os indicadores de origem ecológica, pois compreendem os recursos naturais, como: água, solo, ar, biodiversidade, entre outros (TAVARES; AGRA FILHO, 2008).

Segundo Amaral (2010, p. 24), “dentre as diversas possibilidades de indicadores encontrados na literatura, a Pegada Ecológica apresenta-se como um indicador de sustentabilidade mais voltado para a dimensão ambiental do desenvolvimento sustentável”. A Pegada Ecológica possibilita relacionar o consumo de recursos naturais com a capacidade do ecossistema para produzir recursos e assimilar os resíduos gerados, sinalizando se a biocapacidade já foi extrapolada (TAVARES; AGRA FILHO, 2008).

Para calcular a Pegada Ecológica de um determinado sistema, deve-se, inicialmente, saber quais são os consumos com maior demanda (água, energia, combustível, área construída, papel, alimentos, etc) e, assim, trabalhar com a quantificação mensal ou anual desses insumos, convertendo o uso destes em hectares globais (gha) de terra bioprodutiva. $\mathrm{O}$ gha não é apenas uma unidade de área, mas uma unidade de produção ecológica associada a uma área, cujos resultados podem, também, ser expressos em hectares (WWF, 2012).

A metodologia da Pegada Ecológica (PE), ou Ecological Footprint, foi desenvolvida na década de 90, pelos pesquisadores Mathis Wackernagel e William Rees, da University of British Columbia, publicada no livro "Our Ecological Footprint - Reducing Human Impact on the Earth". Atualmente, o método contábil da Pegada Ecológica é coordenado, hoje, pela Global Footprint Network, fundada em 2003, e suas 50 organizações parceiras (WWF, 2012).

A Pegada Ecológica é um indicador de sustentabilidade que se apoia no conceito da capacidade de suporte com linhas de raciocínio parecidas, porém de metodologias contrárias. A capacidade de suporte estima o número máximo de indivíduos que podem usufruir dos bens de um determinado habitat; a metodologia da Pegada Ecológica calcula o total de área de terra produtiva e de água demandada continuamente para produzir todos os recursos consumidos e para assimilar todos os resíduos produzidos por uma dada população (WACKERNAGEL; REES, 1996). 
Algumas universidades brasileiras e internacionais já utilizam esse indicador na gestão ambiental do campus, conforme apresentado pela Tabela 1 .

\section{Tabela 1 - Pegada Ecológica em diferentes universidades.}

\begin{tabular}{|c|c|c|c|c|}
\hline Universidade & País & Ano & $\begin{array}{c}\text { Recursos } \\
\text { Considerados }\end{array}$ & $\begin{array}{c}\text { Pegada } \\
\text { Ecológica } \\
\left(\text { ha.hab }^{-1} \text {.ano }^{-1}\right)\end{array}$ \\
\hline $\begin{array}{l}\text { Colorado } \\
\text { College }\end{array}$ & $\begin{array}{l}\text { Estados } \\
\text { Unidos }\end{array}$ & 2001 & $\begin{array}{l}\text { Alimentação, energia, } \\
\text { transporte, resíduos, } \\
\text { água e área construída. }\end{array}$ & 2,24 \\
\hline $\begin{array}{l}\text { Universidade de } \\
\text { Toronto }\end{array}$ & Canadá & 2005 & $\begin{array}{l}\text { Alimentação, energia, } \\
\text { transporte, resíduos, } \\
\text { água e área construída. }\end{array}$ & 1,04 \\
\hline $\begin{array}{l}\text { Universidade de } \\
\text { Redlands }\end{array}$ & $\begin{array}{l}\text { Estados } \\
\text { Unidos }\end{array}$ & 1998 & $\begin{array}{l}\text { Energia, Transporte, } \\
\text { resíduos e água. }\end{array}$ & 0,85 \\
\hline $\begin{array}{l}\text { Universidade de } \\
\text { Holme }\end{array}$ & Inglaterra & 2001 & $\begin{array}{l}\text { Alimentação, energia, } \\
\text { transporte, resíduos e } \\
\text { área construída. }\end{array}$ & 0,56 \\
\hline $\begin{array}{c}\text { Universidade } \\
\text { de Santiago de } \\
\text { Compostela }\end{array}$ & Espanha & 2008 & $\begin{array}{l}\text { Energia, transporte, } \\
\text { papel, área construída } \\
\text { e água }\end{array}$ & 0,28 \\
\hline $\begin{array}{l}\text { Universidade de } \\
\text { Newcastle }\end{array}$ & Austrália & 1999 & $\begin{array}{l}\text { Alimentação, energia, } \\
\text { transporte e área } \\
\text { construída. }\end{array}$ & 0,19 \\
\hline $\begin{array}{l}\text { Universidade de } \\
\text { São Paulo }\end{array}$ & Brasil & 2008 & $\begin{array}{l}\text { Água, papel, energia, } \\
\text { transporte e áreas } \\
\text { construídas. }\end{array}$ & 0,19 \\
\hline $\begin{array}{l}\text { Universidade } \\
\text { Federal } \\
\text { Fluminense } \\
\text { - Escola de } \\
\text { Engenharia }\end{array}$ & Brasil & 2013 & Transporte e papel & 0,07 \\
\hline
\end{tabular}

Fonte: Adaptado de USC (2008); Amaral (2010); Nascimento; Lima; Maciel (2013).

Por meio da tabela 1, é fácil notar que o grande diferencial dessa metodologia é a possibilidade de realizar um benchmark ecológico agregador; ou seja, comparar a sustentabilidade entre empresas, eventos, países, cidades ou, como nesse caso, universidades.

Nesse sentido, o objetivo deste trabalho é calcular o tamanho de área bioprodutiva necessária para absorver as emissões de dióxido de carbono $\left(\mathrm{CO}_{2}\right)$, geradas pelas atividades diárias da Universidade Tecnológica Federal do Paraná, Campus Medianeira (UTFPR-MD).

\section{Material e Métodos}

Esta pesquisa segue os conceitos da metodologia original da Pegada Ecológica proposta por Wackernagel e Rees (1996), tendo sido considerada apenas a pegada de carbono, uma vez que os outros componentes, como áreas de cultivo, pastagens, florestas e estoques pesqueiros não possuem relação com a natureza das atividades realizadas em uma universidade. 
A UTFPR-MD ocupa uma área de aproximadamente 12 hectares, localizada no oeste do Estado do Paraná (figura 1), a aproximadamente $580 \mathrm{~km}$ da capital Curitiba.

\section{Figura 1 - Macrolocalização da unidade de estudo (coordenadas 2518'4.66"'S 546'53.06”'W). A) Mapa do Brasil com destaque no Estado do Paraná; B) Mapa do Estado do Paraná com destaque na cidade de Medianeira; C) Mapa da cidade com destaque na unidade de estudo; D) Unidade de estudo destacada em vermelho}

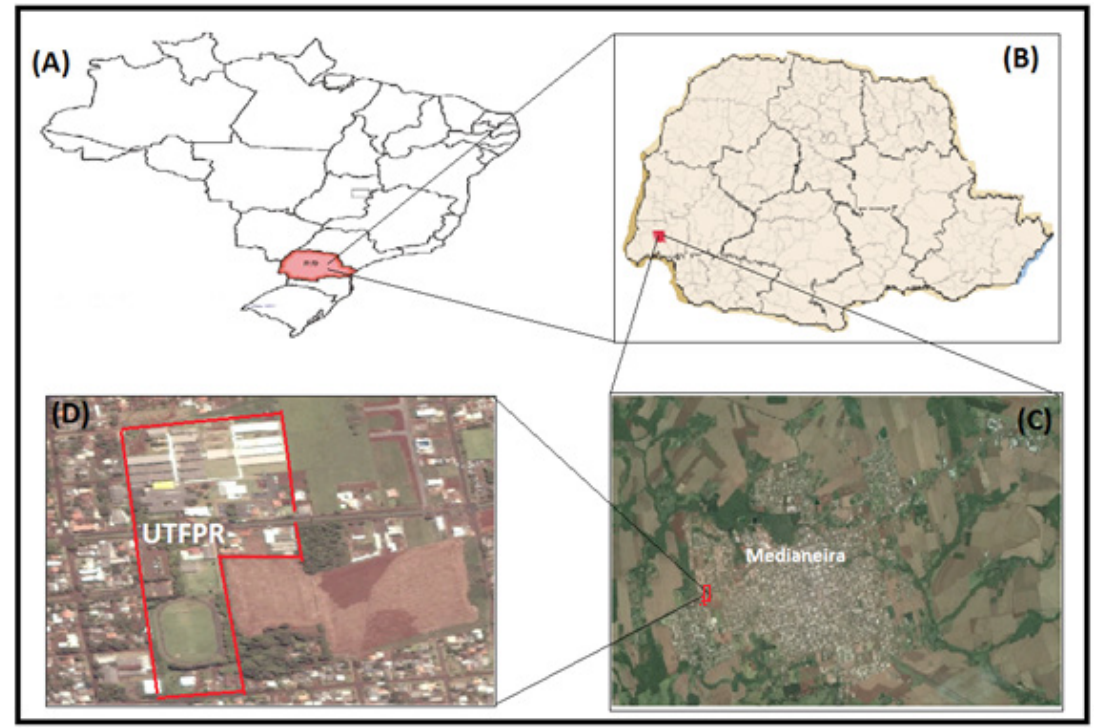

Fonte: GoogleMaps (2015)

A população total deste campus universitário é composta por acadêmicos, professores e servidores. A Tabela 2 apresenta a população total do campus para o $2^{\circ}$ semestre de 2015 .

Tabela 2 - População total da UTFPR-MD.

\begin{tabular}{cc}
\hline Categoria & Quantidade \\
\hline Acadêmicos & 2069 \\
Professores & 188 \\
Servidores & 97 \\
\hline Total & 2354 \\
\hline
\end{tabular}

Fonte: Adaptado de UTFPR (2015).

Para determinar a Pegada Ecológica do UTFPR-MD, buscou-se quantificar o consumo médio dos principais recursos empregados no cotidiano da universidade, durante o ano de 2015.

A estimativa de consumo de alimentos foi baseada na necessidade de compras mensais desses alimentos para suprir a demanda. Para efeito de cálculo anual, estimou-se a quantidade de dias de funcionamento do Restaurante Universitário (RU) no ano, 260 dias (26 dias por mês, 10 meses no ano). Foram considerados os alimentos consumidos durante as refeições de almoço e janta no RU, excluindo-se os lanches e outros alimentos consumidos na cantina do restaurante. 
Com relação ao consumo de combustível, considerou-se somente o utilizado pelos veículos oficiais da universidade. Os registros de consumo de combustível equivalem ao período de janeiro a setembro de 2015 , portanto foi calculada a média para o período de um ano.

A quantificação do consumo de energia elétrica foi estimada com base nos dez primeiros meses do ano, calculando a média e extrapolando-a para doze meses.

No que tange ao aspecto de Infraestrutura e Edifícios, foram enquadradas nesse item todas as áreas construídas ou degradadas, consideradas não produtivas biologicamente, ou seja, que já não têm capacidade de produção de biomassa. Para efeito de cálculo, o fator de emissão considera o tempo de vida útil de um edifício de 50 anos (AMARAL, 2010). Portanto, o valor final de emissão, teve que ser divido por 50, já que o período em estudo é de um ano.

Para o papel foram consideradas neste item somente as folhas de papel sulfite (papel do tipo A4), virgem ou reciclada, adquirido e utilizado pelos diversos setores administrativos, bem como pela empresa que vende materiais escolares, dentro do campus. Com a finalidade de obter a quantidade de papel (em $\mathrm{kg}$ ) multiplicou-se a quantia total de papel utilizado pela massa de uma folha de sulfite, $4,67 \mathrm{~g}$.

Para a adoção do Fator de Emissão, buscou-se apoio nas referências das organizações mais conceituadas nessa temática conforme Quadro 1.

\section{Quadro 1 - Fator de Emissão de cada dado utilizado e respectivas unidades e referências.}

\begin{tabular}{|c|c|c|c|c|}
\hline \multicolumn{2}{|c|}{ Dados Utilizados } & $\begin{array}{l}\text { Fator de } \\
\text { Emissão }\end{array}$ & Unidade & Fonte \\
\hline \multirow{2}{*}{ Alimentos } & Origem Animal & 3,43 & $\mathrm{kgCO}_{2} \cdot \mathrm{kg}$ & \multirow{2}{*}{ MMA (2011) } \\
\hline & Origem Vegetal & 2,10 & Alimento $^{-1}$ & \\
\hline \multicolumn{2}{|c|}{ Energia Elétrica } & 0,1355 & $\mathrm{kgCO}_{2} \cdot \mathrm{kWh}^{-1}$ & MCT (2014) \\
\hline \multicolumn{2}{|c|}{ Infraestrutura e Edifícios } & 520 & $\mathrm{kgCO} \cdot \mathrm{m}^{-2}$ & USC (2008) \\
\hline \multirow{2}{*}{ Papel } & Virgem & 1,84 & \multirow{2}{*}{$\mathrm{kgCO}_{2} \cdot \mathrm{kg}$ papel $^{-1}$} & \multirow{2}{*}{ USC (2008) } \\
\hline & Reciclado & 0,61 & & \\
\hline \multirow{3}{*}{$\begin{array}{l}\text { Mobilidade e } \\
\text { Transporte }\end{array}$} & Diesel & 2,671 & \multirow{3}{*}{$\mathrm{kgCO} \cdot \mathrm{L}^{-1}$} & \multirow{3}{*}{ MMA (2011) } \\
\hline & Etanol & 1,178 & & \\
\hline & Gasolina & 2,269 & & \\
\hline
\end{tabular}

Fonte: USC (2008), MMA (2011), MCT (2014).

A estimativa de emissões de $\mathrm{CO}_{2}$ é obtida através da multiplicação do total consumido de um recurso pelo seu respectivo Fator de Emissão (Equação 1):

Emissão $\left(\mathrm{kgCO}_{2}\right)=$ Consumo (unidade $) *$ Fator de Emissão $\left(\mathrm{kgCO}_{2} /\right.$ unidade $)$

Para realizar o cálculo da Pegada de Carbono, considerou-se o mesmo valor da taxa de absorção de 6,270 t $\mathrm{CO}_{2} \cdot$ ha $^{-1}$. ano $^{-1}$, cujo valor é o mesmo utilizado em outro estudo de cálculo de Pegada Ecológica em contextos universitários, na Universidade de Santiago de Compostela (USC), realizado por Rodriguez; Iglesias e Álvarez (2009) e na Universidade de São Paulo - São Carlos por Amaral (2010). 
Para obter a Pegada Ecológica, que é o total de área bioprodutiva necessária para absorver as emissões de $\mathrm{CO}_{2}$, multiplica-se o total emitido pela Taxa de Absorção supracitada, de acordo com Equação 2:

Área $(\mathrm{ha})=$ Emissão $\left(\mathrm{kgCO}_{2}\right) /$ Taxa de Absorção $\left(\mathrm{kgCO}_{2} \cdot h a^{-1} \cdot a n o^{-1}\right)$

\section{Resultados e Discussão}

No Restaurante Universitário (RU), são servidas, em média, 1100 refeições diariamente, sendo 700 no almoço e 400 no jantar. São fornecidos basicamente 16 itens alimentares, distribuídos de forma variável de acordo com o cardápio diário. As quantidades de alimentos consumidos per capita estão discriminadas na Tabela 3.

\section{Tabela 3 - Quantidade per capita de alimentos consumidos por pessoa no RU.}

\begin{tabular}{cccc}
\hline $\begin{array}{c}\text { Tipo de } \\
\text { Alimento }\end{array}$ & $\begin{array}{c}\text { Quantidade } \\
\left(\mathbf{k g} \cdot \text { per capita }^{-\mathbf{1}} \text {.dia }^{-\mathbf{1}}\right)\end{array}$ & $\begin{array}{c}\text { Tipo de } \\
\text { Alimento }\end{array}$ & $\begin{array}{c}\text { Quantidade } \\
\left(\mathbf{k g} \cdot \text { per capita }^{-1} \mathbf{. d i a}^{-\mathbf{1}} \mathbf{)}\right.\end{array}$ \\
\hline Arroz & 0,070 & Bife suíno & 0,050 \\
Feijão & 0,055 & $\begin{array}{c}\text { Almôndega } \\
\text { Estrogonofe de }\end{array}$ & 0,035 \\
Purê de batata & 0,030 & carne & 0,040 \\
Macarrão & 0,025 & Alface & 0,005 \\
Polenta & 0,015 & Pepino & 0,010 \\
Mandioca & 0,013 & Beterraba & 0,015 \\
Frango grelhado & 0,080 & Cenoura & 0,015 \\
Frango assado & 0,100 & Repolho & 0,020 \\
\hline
\end{tabular}

Fonte: Autores (2015).

Considerando que os fatores de emissões são diferentes para alimentos de origem animal e vegetal, obteve-se a média per capita diária de consumo por origem de alimento. Com base no consumo diário per capita de alimentos, pôde-se calcular as respectivas emissões, bem como a Pegada Ecológica individual descrita na Tabela 4.

\section{Tabela 4 - Emissão (t $\mathrm{CO}_{2}$ ) e PE per capita do consumo de alimentos na UTFPR-MD}

\begin{tabular}{|c|c|c|c|}
\hline Origem do Alimento & $\begin{array}{c}\text { Quantidade } \\
\left(\text { kg.per capita }{ }^{-1} \cdot \text { dia }^{-1}\right)\end{array}$ & $\begin{array}{c}\text { Emissão } \\
\left(\mathrm{t} \mathrm{CO}_{2} \cdot \text { per capita }^{-1}\right)\end{array}$ & $\begin{array}{l}\text { Pegada Ecológica } \\
\left.\text { (gha.per capita }^{-1}\right)\end{array}$ \\
\hline Animal & 0,305 & 0,271 & 0,0433 \\
\hline Vegetal & 0,273 & 0,149 & 0,0237 \\
\hline Total & 0,578 & 0,421 & 0,0670 \\
\hline
\end{tabular}

Fonte: Autores (2015). 
Os resultados obtidos apontam que cada pessoa que almoça ou janta no restaurante universitário consome, em média, 0,578 kg de alimentos. Cerca de 52,7\% desses alimentos são de origem animal. Esse consumo de alimentos per capita, no período de um ano, é responsável pela emissão de quase meia tonelada de $\mathrm{CO}_{2}$, enquanto que a Pegada Ecológica individual para o consumo de alimentos no campus é de 0,06 gha.per capita-1 ${ }^{-1}$ ou seja, representa uma área de $670 \mathrm{~m}^{2}$.

A PE total é resultado da multiplicação da PE per capita pela quantidade de refeições servidas durante todo o ano (Figura 2).

\section{Figura 2 - Emissões de $\mathrm{CO}_{2}$ e Pegada Ecológica de alimentos da UTFPR-MD.}

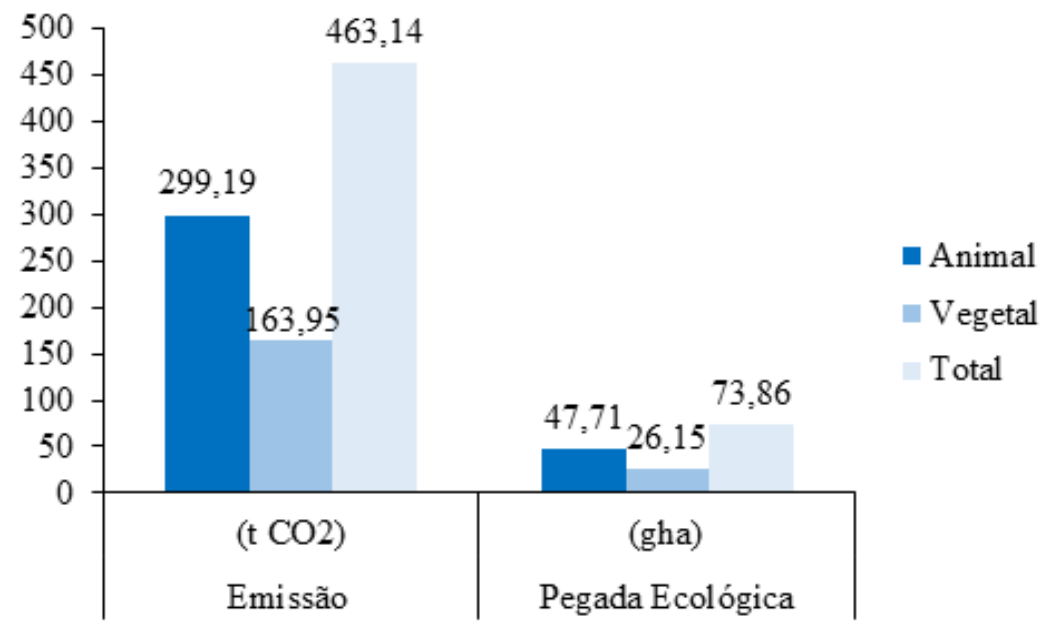

Fonte: Autores (2015).

Mesmo havendo certo equilíbrio no consumo de alimentos de origem animal e vegetal, como observado na Tabela 4, as emissões de $\mathrm{CO}_{2}$ e a Pegada Ecológica do consumo de alimentos de origem animal representa quase o dobro dos de origem vegetal, isso se deve ao fato de que os alimentos de origem animal, em sua grande maioria, são geradores de $\mathrm{CO}_{2}$ em grande parte do seu ciclo de vida.

O consumo de alimentos no RU da UTFPR-MD gera $463.14 \mathrm{~kg} \mathrm{CO}$. $\mathrm{ano}^{-1}$, de modo a resultar em uma Pegada Ecológica de 73,86 gha, essa PE corresponde a aproximadamente 6 vezes a área do campus.

O campus possui uma frota de nove veículos oficiais que utilizam os seguintes combustíveis: diesel, etanol ou gasolina (Tabela 5). 


\section{Tabela 5 - Veículos oficiais do campus e combustíveis utilizados.}

Veículo Tipo de Combustível Consumo de Combustivel (L)

\begin{tabular}{ccc}
\hline Ford Ranger & Diesel & 2542,11 \\
\hline \multirow{2}{*}{ Sprinter } & Diesel & 4415,6 \\
\hline \multirow{2}{*}{ Fiat Uno } & Etanol & 220,6 \\
\hline \multirow{2}{*}{ Saveiro } & Etanol & 198,21 \\
\cline { 2 - 3 } & Gasolina & 84,95 \\
\hline \multirow{2}{*}{ Astra } & Etanol & 1180,86 \\
\hline \multirow{2}{*}{ Astra } & Gasolina & 506,08 \\
\cline { 2 - 3 } & Etanol & 1490,42 \\
\hline \multirow{2}{*}{ Cruze } & Gasolina & 638,75 \\
\hline \multirow{2}{*}{ Ducato } & Etanol & 2176,36 \\
\cline { 2 - 3 } & Gasolina & 932,74 \\
\hline \multirow{2}{*}{ Fluence } & Etanol & 1503,8 \\
\cline { 2 - 3 } & Gasolina & 644,48 \\
\hline
\end{tabular}

Fonte: Autores (2015).

Apenas dois veículos utilizam óleo diesel, porém são os que apresentam os maiores valores de consumo de combustível dentre todos veículos avaliados.

O veículo Fiat Uno utiliza exclusivamente etanol e, aparentemente, é o menos utilizado da frota de veículos, visto que o consumo de combustível está muito abaixo dos demais. Os outros seis veículos são da categoria Flex, que utilizam etanol ou gasolina. As emissões de $\mathrm{CO}_{2}$ e a Pegada Ecológica de cada veículo estão apresentadas na Figura 3.

Quanto à sustentabilidade dos veículos, destaca-se a diferença entre as emissões de $\mathrm{CO}_{2}$ e PE dos veículos Sprinter e Ducato. Estes são veículos parecidos, utilizados para grandes quantidades de passageiros e longas distâncias, no entanto a diferença no tipo de combustível utilizado faz com que a Sprinter gere impactos maiores. Nesse contexto, sugere-se que haja um equilíbrio na utilização desses dois automóveis. 


\section{Figura 3 - Emissões de $\mathrm{CO}_{2}$ e Pegada Ecológica de cada veículo oficial do campus.}

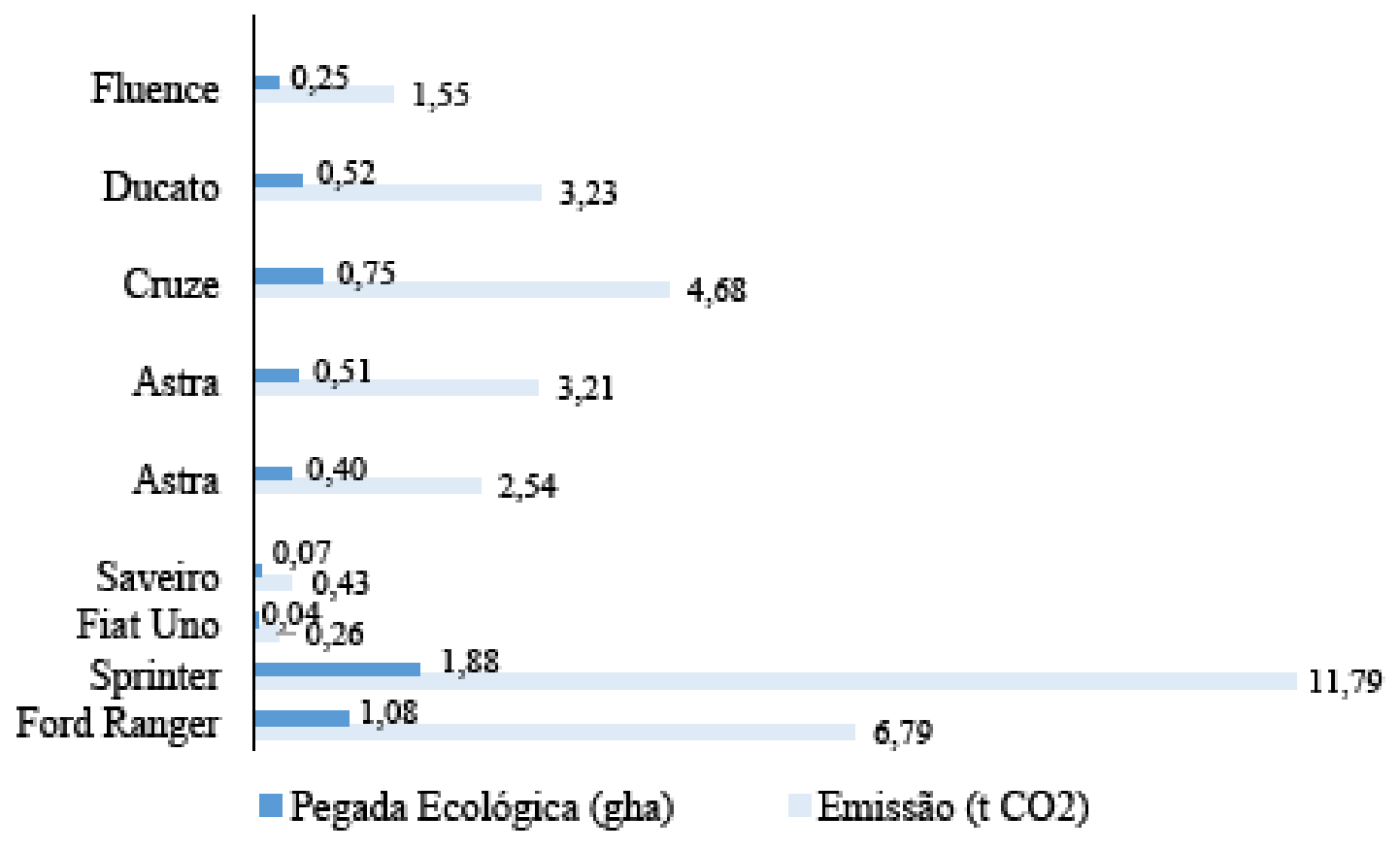

Fonte: Autores (2015).

O consumo total de combustível, bem como suas respectivas emissões de $\mathrm{CO}_{2}$ e Pegada Ecológica estão descritos na Tabela 6.

\section{Tabela 6 - Total de Consumo, Emissões de $\mathrm{CO}_{2}$ e Pegada Ecológica por tipo de combustível no ano de 2015.}

\begin{tabular}{cccc}
\hline Combustivel & Consumo (L) & $\begin{array}{c}\text { Emissão } \\
(\mathbf{t ~ C O})\end{array}$ & $\begin{array}{c}\text { Pegada Ecológica } \\
(\text { gha) }\end{array}$ \\
\hline Diesel & $6.957,71$ & 18,58 & 2,96 \\
Etanol & $7.491,15$ & 8,82 & 1,41 \\
Gasolina & $3.115,95$ & 7,07 & 1,13 \\
Total & $17.564,81$ & 34,47 & 5,50 \\
\hline
\end{tabular}

Fonte: Autores (2015).

O combustível mais utilizado pelo setor de transporte do campus é o etanol, com mais de 7 mil litros. Esse valor representa $42 \%$ do consumo total dos três tipos de combustíveis.

O consumo de diesel, apesar de ser utilizado por apenas dois veículos é predominante na composição das emissões e PE, representa 39\% do total. Enquanto que a gasolina representa apenas $17 \%$ do total, essa baixa representatividade se justifica pelo fato de os veículos serem flex e, consequentemente, abastecidos com etanol com mais frequência, devido à viabilidade econômica. Por fim, o consumo de combustível pelos 9 veículos oficiais da UTFPR-MD emite, por ano, 34,47 t $\mathrm{CO}_{2}$, resultando em uma Pegada Ecológica de 5,50 gha. 
A estimativa obtida do consumo total de energia elétrica no campus, para o ano de 2015, é de 1.000.895 kWh. Portanto, tem-se uma média de 83.407 kWh.mês ${ }^{-1}$, aproximadamente.

\section{Tabela 7 - Consumo, Emissão de $\mathrm{CO}_{2}$ e PE per capita de energia elétrica do câmpus.}

\begin{tabular}{cccc}
\hline Recurso & $\begin{array}{c}\text { Consumo } \\
(\mathbf{k W h} \cdot \text { per capita }\end{array}$ & $\begin{array}{c}\text { Emissão } \\
\left(\mathbf{t ~} \mathbf{C O}_{2} \cdot \text { per capita }^{-\mathbf{1}}\right)\end{array}$ & $\begin{array}{c}\text { Pegada Ecológica } \\
\left.\text { (gha.per capita }^{-1}\right)\end{array}$ \\
\hline Energia Elétrica & 425,18 & 0,576 & 0,0092 \\
\hline
\end{tabular}

Fonte: Autores (2015).

O consumo per capita de energia elétrica anual é de 425,18 KWh (Tabela 7). Esse consumo gera cerca de meia tonelada per capita de $\mathrm{CO}_{2}$, resultando na Pegada Ecológica de $92 \mathrm{~m}^{2}$.per capita $^{-1}$ ou 0,0092 gha.per capita ${ }^{-1}$.

A quantificação total das emissões de $\mathrm{CO}_{2}$ e a Pegada Ecológica deste recurso estão apontadas na Tabela 8.

\section{Tabela 8 - Emissão de $\mathrm{CO}_{2}$ e Pegada Ecológica total de energia elétrica do campus.}

\begin{tabular}{cccc}
\hline Recurso & $\begin{array}{c}\text { Consumo } \\
(\mathbf{k W h})\end{array}$ & $\begin{array}{c}\text { Emissão } \\
(\mathbf{t ~ C O})\end{array}$ & $\begin{array}{c}\text { Pegada Ecológica } \\
\mathbf{( g h a )}\end{array}$ \\
\hline Energia Elétrica & 1.000 .895 & 135,62 & 21,63
\end{tabular}

Fonte: Autores (2015)

O consumo de energia elétrica da UTFPR-MD resulta na emissão por ano 135,62 t CO, o que corresponde a uma Pegada Ecológica de 21,63 gha, área equivalente a quase o dobro do tamanho do câmpus.

A UTFPR-MD possui uma área total de 122.165,60 $\mathrm{m}^{2}$, cerca de 12,22 hectares, a composição do terreno está descrita na tabela 9.

\section{Tabela 9 - Composição do terreno da UTFPR-MD.}

\begin{tabular}{ccc}
\hline Área total $\left(\mathbf{m}^{\mathbf{2}}\right)$ & Área construída $\left(\mathbf{m}^{\mathbf{2}}\right)$ & Área construída coberta $\left(\mathbf{m}^{\mathbf{2}}\right)$ \\
\hline $122.165,60$ & $32.291,00$ & $12.916,51$ \\
\hline
\end{tabular}

Fonte: Autores (2015).

O terreno possui 45.207,51 $\mathrm{m}^{2}$ de área construída e/ou coberta, portanto não bioprodutiva para efeito de cálculo da PE. Essa área representa 37\% da área total do campus.

As emissões de $\mathrm{CO}_{2}$ e a Pegada Ecológica deste recurso encontram-se na Tabela 10. 


\section{Tabela 10 - Emissão de $\mathrm{CO}_{2}$ e Pegada Ecológica total das áreas construídas do campus.}

\begin{tabular}{cccc}
\hline Recurso & $\begin{array}{c}\text { Área Improdutiva } \\
\left(\mathbf{m}^{\mathbf{2}}\right)\end{array}$ & $\begin{array}{c}\text { Emissão } \\
(\mathbf{t ~ C O} \mathbf{2})\end{array}$ & $\begin{array}{c}\text { Pegada Ecológica } \\
\mathbf{( g h a )}\end{array}$ \\
\hline Área Construída & $45.207,51$ & 470,15 & 75,00 \\
\hline
\end{tabular}

Fonte: Autores (2015).

No cenário atual, as emissões anuais de $\mathrm{CO}_{2}$ são de 470 toneladas, o que representa uma Pegada Ecológica de 75 gha. Porém, essa pegada tende aumentar com a tendência de expansão do csmpus que nos, últimos 5 anos, construiu 5 novos blocos de sala de aula e laboratórios, novo edifício para biblioteca e expandiu sua área total.

Considerou-se duas fontes fornecedoras de papel na universidade: os papéis adquiridos pela própria universidade para utilização nos setores administrativos, em geral, e os papéis comercializados pela empresa "Papelaria Universitária", responsável pelos serviços de xerox, impressões, etc.

Com base nos dados de consumo fornecidos, foi elaborada a Tabela 11, que apresenta a quantidade de papel consumida no ano de 2015.

\section{Tabela 11 - Quantidade de papel consumida na UTFPR-MD.}

\begin{tabular}{ccc}
\hline Setor & Tipo de papel & Quantidade (folha A4) \\
\cline { 2 - 3 } Universidade em geral & Virgem & 400.000 \\
& Reciclado & 10.000 \\
Papelaria Universitária & Virgem & 630.000 \\
& Reciclado & Não consta \\
\hline Total & & 1.040 .000 \\
\hline
\end{tabular}

Fonte: Autores (2015).

Constatou-se um consumo anual de mais de 1 milhão de folhas A4, o que equivale a cerca de 5 mil kg de folhas, sendo que apenas 10.000 folhas são do tipo recicladas, esse valor representa menos de $1 \%$ do total. Assim, as emissões de $\mathrm{CO}_{2}$ e a Pegada Ecológica geradas pelo consumo de papel sulfite em todo o campus encontram-se discriminadas na Tabela 12.

Tabela 12 - Emissão de $\mathrm{CO}_{2}$ e Pegada Ecológica do consumo de papel.

\begin{tabular}{cccc}
\hline Tipo de Papel & $\begin{array}{c}\text { Quantidade } \\
\text { (folha A4) }\end{array}$ & $\begin{array}{c}\text { Emissão } \\
\left(\mathrm{t} \mathrm{CO}_{2}\right)\end{array}$ & $\begin{array}{l}\text { Pegada Ecológica } \\
\text { (gha) }\end{array}$ \\
\hline Virgem & 1.030 .000 & 8,8506 & 1,4116 \\
Reciclado & 10.000 & 0,0285 & 0,0045 \\
\hline Total & 1.040 .000 & 8,8791 & 1,4161
\end{tabular}

Fonte: Autores (2015).

Para o consumo de papel, as emissões anuais de $\mathrm{CO}_{2}$ são de 8,87 toneladas, o que representa uma Pegada Ecológica de 1,4 gha, equivalente a uma área de aproximadamente $11 \%$ da área do campus. 
A Pegada Ecológica total do campus é composta pelos cinco recursos apresentados anteriormente. A avaliação da composição global do indicador está representada na Tabela 13.

\begin{tabular}{ccc} 
Tabela 13 - Composição da Pegada Ecológica total da UTFPR-MD. \\
\hline Recurso & Emissão (t CO $\mathbf{2})$ & Pegada Ecológica (gha) \\
\hline Consumo de alimentos & 463,14 & 73,86 \\
Mobilidade e transporte & 34,47 & 5,50 \\
Consumo de energia elétrica & 135,62 & 21,63 \\
Infraestrutura e edifícios & 470,15 & 75,00 \\
Consumo de papel & 8,88 & 1,42 \\
\hline Total & 1112,26 & 177,41
\end{tabular}

Fonte: Autores (2015).

Para uma melhor discussão e compreensão dos resultados acima apresentados, elaborou-se a Figura 4, que ilustra a participação relativa (em \%) de cada recurso considerado na composição desta Pegada Ecológica.

Figura 4 - Composição da Pegada Ecológica da UTFPR-MD.

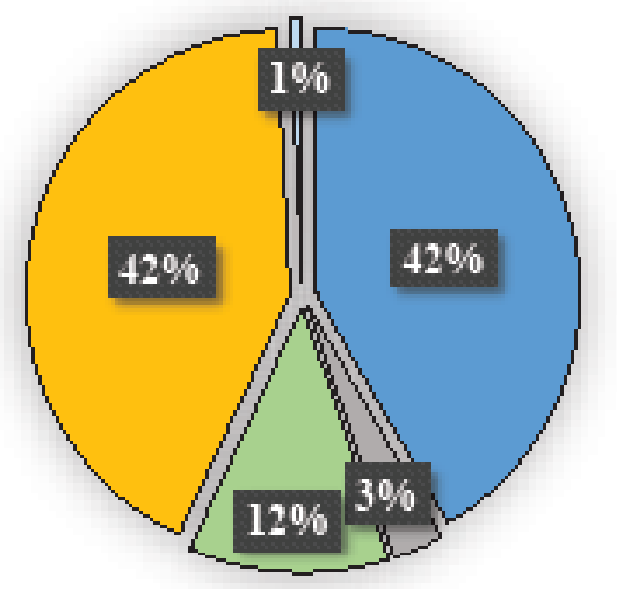

口Consumo de Alimentos

$\square$ Mobilidade e Transporte

$\square$ Consumo de Energia Elétrica

口Infraestrutura e Edificios

$\square$ Consumo de Papel

Fonte: Autores (2015).

O somatório das pegadas de todos os recursos em estudo resultou na Pegada Ecológica de 177,41 gha, cujo valor representa uma área 14,5 vezes maior que o próprio campus. No entanto, a $\mathrm{PE}$ per capita total é 0,0753 gha.ano $^{-1}$, esse valor está próximo à $\mathrm{PE}$ nacional que é 0,0725 gha. hab $^{-1} \cdot$ ano $^{-1}$ (GLOBAL FOOTPRINT NETWORK, 2011).

Dentre o valor total da Pegada Ecológica do campus, em 2015, destaca-se o consumo de Alimentos e Infraestrutura e Edifícios, juntos correspondem a $84 \%$ das emissões de $\mathrm{CO}_{2}$ e da composição da PE. Como medida mitigadora nesses dois fatores, considera-se importante a apresentação desses resultados para a comunidade acadêmica do campus, principalmente aos que fazem refeições no Restaurante Universitário visando à sensibilização para a redução de desperdício de alimentos. Quanto à Infraestrutura e Edificações, embora não se possa evitar o crescimento da universidade, sugere-se a otimização dos espaços já construídos.

O levantamento dos itens Consumo de Papel e de Mobilidade e Transporte indicam que esses são os únicos que apresentam PE menor que a própria área do campus, que é de 12,22 hectares (Figura 5). No entanto, em estudos futuros, esses valores podem apresentar resultados 
mais expressivos se forem levados em conta a mobilidade e consumo de papel direto por parte dos acadêmicos.

A fim de facilitar o entendimento das diferenças de áreas por cada Pegada Ecológica e, também, para compará-las com a área do campus, elaborou-se a Figura 5.

\section{Figura 5 - Comparação de áreas da Pegada Ecológica com a área do campus.}
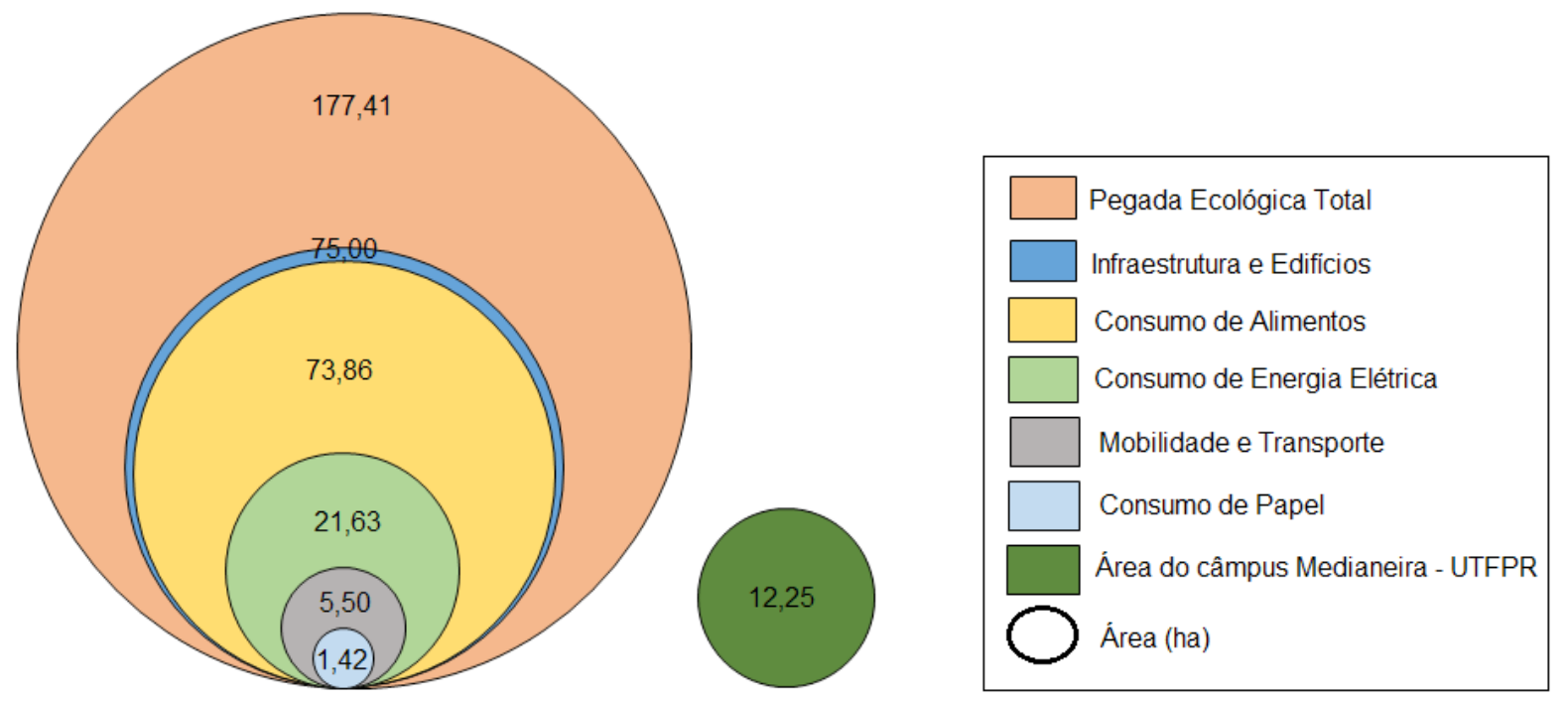

Fonte: Autores (2015).

$\mathrm{O}$ consumo de energia elétrica resultou na $\mathrm{PE}$ com uma área quase duas vezes maior que a área total do campus. Essa constatação por si só, pode fornecer fundamento para o desenvolvimento de projetos de redução de energia ou até mesmo de utilização de fontes alternativas.

Como a Pegada Ecológica é um indicador ambiental que trabalha com mensuração e estimativas, as áreas resultantes dos cálculos não apresentam exatidão, embora auxiliam na avaliação da sustentabilidade ou insustentabilidade do local em estudo.

Nesse contexto, os resultados encontrados indicam que somente o consumo de papel e combustível apresentam sustentabilidade, uma vez que as áreas encontradas são menores que a área do campus. Os outros recursos considerados, consumo de energia, alimentos e área construída, por sua vez, apresentam insustentabilidade, já que as áreas necessárias para absorver suas emissões de $\mathrm{CO}_{2}$ são duas e seis vezes maiores, respectivamente, que a área do campus.

\subsection{Comparação da Pegada Ecológica entre Universidades}

A comparação dos resultados da UTFPR-MD com o campus São Carlos da Universidade de São Paulo (USP) (AMARAL, 2010) e Universidade de Santiago de Compostela (USC, 2008) da Espanha está expressa na Tabela 14. 


\section{Tabela 14 - Comparação da Pegada Ecológica em diferentes campus universitários.}

\begin{tabular}{cccc}
\hline \multirow{2}{*}{ Recurso } & \multicolumn{3}{c}{ Pegada Ecológica (gha.ano $^{-1}$ ) } \\
& UTFPR-MD & $\begin{array}{c}\text { USP -São } \\
\text { Carlos }\end{array}$ & $\begin{array}{c}\text { USC- } \\
\text { Espanha }\end{array}$ \\
\hline Consumo de energia elétrica & 21,63 & $1.027,49$ & 3285,49 \\
Mobilidade e transporte & 5,5 & 197,34 & 917,07 \\
Consumo de papel & 1,42 & 18,39 & 69,9 \\
Infraestrutura e Edifícios & 75 & 288,17 & 802,03 \\
Consumo de alimentos & 73,86 & Não incluso & Não incluso \\
Consumo de água & Não incluso & 12,88 & 27,14 \\
\hline Total & 177,41 & $1.524,37$ & $5.101,62$ \\
\hline População & 2354 & 8023 & 32600 \\
\hline PE per capita & 0,07 & 0,19 & 0,16 \\
\hline
\end{tabular}

Fonte: Autores (2015).

Na USP São Carlos e na USC, nota-se que o consumo de água está entre os recursos considerados, diferentemente da UTFPR-MD, pois "a pegada ecológica da água é baseada na energia necessária para extrair, tratar e abastecer a água” (CARMO, 2008, p.27). Contudo, o consumo de água, embora seja um fator relevante quando se trata de sustentabilidade, não foi considerado nesta pesquisa visto que toda água utilizada neste campus universitário é captada de poços artesianos, logo não passam pela etapa de tratamento.

A comparação da PE total da UTFPR-MD com as outras duas universidades indica que esse é o campus mais sustentável. Porém, essa comparação não deve ser feita analisando exclusivamente os valores brutos da $\mathrm{PE}$ de cada recurso, tampouco o total, uma vez que a sustentabilidade está atrelada ao estilo de vida e consumo de recursos de cada membro que compõem a população do objeto de estudo. Essa afirmação pode ser confirmada com a comparação dos resultados da $\mathrm{PE}$ da USP e USC, onde a USC mesmo apresentando maior pegada em todos os itens considerados e ainda uma população muito maior que a USP, ela consegue ter uma PE per capita menor o que indica um padrão de consumo mais harmônico com o meio ambiente. Desta forma, a avaliação de sustentabilidade do estudo deve ser realizada em função da PE per capita dos 3 campus universitários.

Partindo dessa premissa, observa-se que a UTFPR-MD apresenta maior sustentabilidade que os outros dois, pois sua PE per capita é 63\% menor que USP - São Carlos e 56\% menor que da USC. Embora seja um aspecto positivo, é fundamental diminuir ainda mais esse índice, visto que é necessária uma área 15 vezes maior do que o próprio câmpus para absorver mais de mil toneladas anuais de $\mathrm{CO}_{2}$ geradas indiretamente pelas atividades do cotidiano da UTFPR-MD.

\section{Conclusões}

O campus UTFPR-MD apresentou menor índice per capita de PE, ou seja, maior sustentabilidade em relação a outras universidades. Embora seja um ponto positivo, a PE total do campus equivale a uma área 15 vezes maior do que o tamanho da própria universidade para absorver todo o $\mathrm{CO}_{2}$, gerado indiretamente pelas atividades do cotidiano da universidade. Entende-se que seja algo natural essa relação de déficit de área, uma vez que o atual padrão de

59nsumo e estilo de vida dificultam um cenário inverso.
Ambiência - Revista do Setor de Ciências Agrárias e Ambientais V.15 N.1 Jan/Abr 2019 
A identificação dos recursos que possuem maiores potenciais para geração de impactos ambientais, no que diz respeito ao consumo foi fundamental no presente estudo, pois quanto maior a quantidade de recursos considerados mais fiel à realidade serão os resultados.

Nesse sentido, ressalta-se a importância da Pegada Ecológica como uma ferramenta transversal que, devido à facilidade de aplicação e compreensão dos resultados, como nesta pesquisa, surge como peça fundamental na gestão ambiental do campus, podendo atuar como parte de um sistema de melhoria contínua, mensurando, avaliando e, consequentemente, reduzindo os índices de insustentabilidade.

\section{Referências}

AMARAL, R. R. Análise da aplicabilidade da Pegada Ecológica em contextos universitários: estudo de caso no câmpus de São Carlos da Universidade de São Paulo. 110 f. Monografia (Graduação em Engenharia Ambiental) - Escola de Engenharia de São Carlos, Universidade de São Paulo, 2010.

ANDRADE, B.; VAN BELLEN, H. M. Turismo e Sustentabilidade no Município de Florianópolis: Uma avaliação a Partir do Método da Pegada Ecológica. In: XXX Encontro da ANPAD, 23 a 27 de setembro - Salvador/BA. Anais... Salvador/BA, 2006.

CARMO, A. O. do. Pegada ecológica: possibilidades e limitações a partir de sua aplicação para a cidade do Salvador-BA. 170 f. Dissertação (Mestrado em Engenharia Ambiental Urbana), Universidade Federal da Bahia, Salvador, 2008

DAILY, G. C.; EHRLICH, P. R. Population, Sustainability, and Earth's Carryng Capacity. A framework for estimating population size and lifestyles that could be sustained withot undermining future generations. Bioscience, v. 42, n 10, p.761-771, nov. 1992.

GLOBAL FOOTPRINT NETWORK. Ecological Wealth of Nations. Disponível em: < http://www.footprintnetwork.org/ecological_footprint_nations/biocapacity_per_capita. html>. Acesso em: 22.out.2015.

LEITE, A. M. F; VIANA, M. O. L. Pegada Ecológica: instrumento de análise do metabolismo do sócio-ecossistema urbano. Fortaleza: IBAMA, PRODEMA, UFC, 2001.

MADURO-ABREU, A.; NASCIMENTO, D. T; MACHADO, L. O.R; COSTA, H. A. Os limites da Pegada Ecológica. Desenvolvimento e Meio Ambiente, Brasília, n.19, p.73-87, 2009. Editora UFPR.

MINISTÉRIO DE CIÊNCIA ETECNOLOGIA (MCT). Fator de Emissão de CO2 para utilizações que necessitam do fator médio de emissão do Sistema Interligado Nacional do Brasil. Disponível em: < http://www.mct.gov.br/index.php/content/ view/321144.html>. Acesso em: 01.nov.2015 
MINISTÉRIO DO MEIO AMBIENTE (MMA). Introdução à Pegada de Carbono. Out.2011. 27 p. Disponível em:<http://www.mma.gov.br/estruturas/255/_arquivos/3_ introducton_to_carbon_footprinting_255.pdf >. Acesso em: 13.out.2015

MONTEIRO, M. C. A.A Qualidade e o Impacto no Meio Ambiente. 66 f. Monografia (Especialização em Administração da Qualidade), Universidade Candido Mendes, Rio de Janeiro, 2010.

NASCIMENTO, S. C; LIMA, G. B.A; MACIEL, G. F. S. Aplicação da Ferramenta Pegada Ecológica para Análise de Impactos Ambientais Gerados pelas Atividades Acadêmicas de Alunos da Escola de Engenharia da Universidade Federal Fluminense. In: XXX ENCONTRO NACIONAL DE ENGENHARIA DE PRODUÇÃO. Out. 2013, Salvador.

PEREIRA, L. G. Síntese dos métodos de Pegada Ecológica e Análise Emergética para diagnóstico da sustentabilidade de países: o Brasil como estudo de caso. $183 \mathrm{f}$. Dissertação (Mestrado em Engenharia de Alimentos) - Faculdade de Engenharia de Alimentos, Universidade Estadual de Campinas, Campinas, 2008.

TAVARES, A. O. C.; AGRA FILHO, S. S. Aplicações da Pegada Ecológica no Brasil: um estudo comparativo. Revista Brasileira de Ciências Ambientais. São Paulo v. 21, p. 54-64, set. 2011.

UNIVERSIDADE DE SANTIAGO DE COMPOSTELA (USC).Impacto Ambiental en Centros da USC. Fev. 2008. Disponível em:< https://www.usc.es/plands/seccions/ datos_plan/eixe2/pegada_ecoloxica/estudo/estudo_pdf/impacto\%20ambiental\%20 final\%20web-6-6-08.pdf>. Acesso em: 20 set. 2014.

UNIVERSIDADE TECNOLÓGICA FEDERAL DO PARANÁ (UTFPR). Mapa com todos os câmpus da UTFPR. Disponível em: <http://www.utfpr.edu.br/estruturauniversitaria/diretorias-de-gestao/dircom/design/mapa-parana-com-todos-os-campusda-utfpr/view> Acesso em: 08 dez. 2014.

WACKERNAGEL, M.; REES, W. Our ecological footprint: reducing human impact of the Earth. 6 ed. Canada: New Society Publisher, 1996.

WORLD WILDLIFE FUND (WWF). A Pegada Ecológica de Campo Grande e a Família das Pegadas. Campo Grande, 2012.127 p. 